\title{
A survey-based study on misplaced or damaged removable retainers
}

\begin{abstract}
Retention is one of the most important challenges faced by the orthodontist. Patient's need to be reminded that retention phase is a continuation of orthodontic treatment and preserving treatment results successfully will depend on their compliance towards wearing and maintaining appropriate appliances. ${ }^{1}$ The Retention plan among clinicians can vary from removable or fixed appliances, but the most common regime has been an upper removable appliance and a lower fixed appliance. ${ }^{2}$ The removable retainers are often prescribed for full time wear for the first 6 months. Patients often return to the dental office requesting new retainers due to damage or because they lost them. We did a study to identify the most common ways removable retainers are lost or damaged and how patients can be educated during delivery of removable retainers to avoid these causes. ${ }^{3}$
\end{abstract}

Volume 7 Issue 5 - 2017

Pratik Premjani

Specialist Orthodontist, European University College (previously Nicolas \& Asp University College), UAE

\author{
Correspondence: Pratik Premjani, Specialist Orthodontist, \\ European University College (previously Nicolas \& Asp \\ University College), Dubai Healthcare City, Ibn Sina Building, No \\ 27, Block D, 3rd Floor, Office 302, PO Box 53382, Dubai, UAE, \\ Tel 97| 4362 4788, Fax 97| 4362 4793, \\ Email pratik.premjani@gmail.com
}

Received: June 24, 2017 | Published: July 25, 2017

\section{Materials and methods}

A study was designed to include the next 200 consecutive patients who walked in the Department of Orthodontics at European University College requesting new retainers. Only patients who damaged or misplaced their retainers were included and were asked to fill a questionnaire (Figures 1-3).

\section{Results}

\section{Gender}

Male- 143

Female -67

Name :

File Number :

Q1) When did you finish your fixed orthodontic treatment?

Q2) Which kind of retainer were you wearing?

A) Hawley type retainer

B) Clear vacuum formed retainer

C) Other:
Q3) Reason for new retainer.
a) Misplaced/ Lost
b) Damaged

Q4) How did you misplace/damage your retainer?

\begin{abstract}
Q5) Were you carrying the case provided (retainer box) when the incident took place? a) Yes b) No
\end{abstract}

Q6) Did you immediately contact the dental office when you $\begin{array}{lll}\text { misplaced/damaged your retainer? } & \text { a) Yes b) No }\end{array}$

Q7) If Answer to Q6) is b) No, then after how long did you contact the dental office?

Figure I Survey Form given to patients who lost or damaged their retainers. 


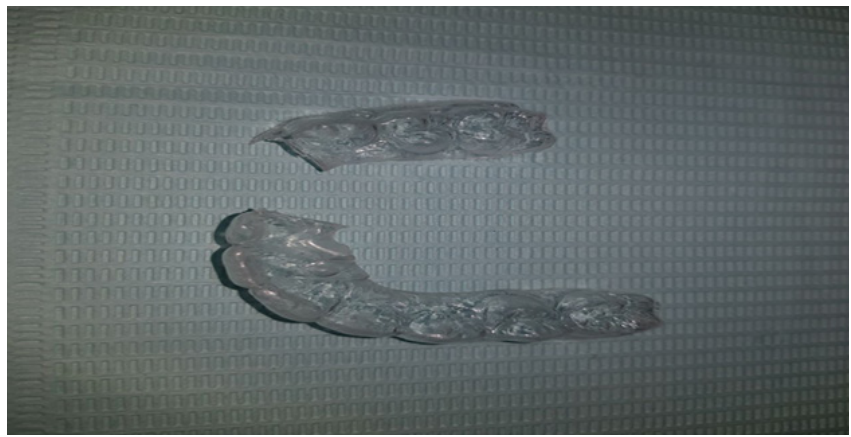

Figure 2

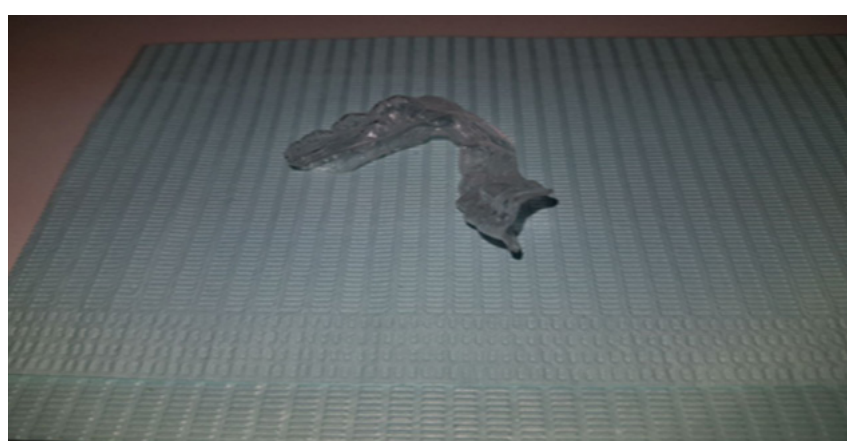

Figure 3

\section{Type of removable retainers lost/damaged}

169 upper and 3 lower clear vacuum type retainers compared to 24 upper and 4 lower Hawley type retainers upper.

1. Upper vacuum type clear retainer- 169

2. Lower vacuum type clear retainer- 3

3. Upper Hawley type clear retainer- 24

4. Lower Hawley type clear retainer- 4

\section{Time in retention phase (Table I)}

Table I Time in retention phase

\begin{tabular}{ll}
\hline Time Since Debonding & No. of Patients \\
\hline Less than I week & 58 \\
More than I week to I month & 24 \\
Between I month and 3 months & 19 \\
Between 3 months and 6 months & 8 \\
Between 6 months and I year & 15 \\
Between I year and 2 years & 43 \\
More than 2 years & 33 \\
\hline
\end{tabular}

\section{Damaged or Misplaced}

29 retainers were damaged, while 171 retainers were lost. All 29 of the damaged retainers were clear vacuum type retainers (essix).

\section{Discussion}

In our study we found that more 'clear vacuum formed type retainer' were lost when compared to Hawley retainers, this could be due to the fact that these clear retainers often times cannot be spotted by others, making it more prone to losing it. A majority of the patients (133 out of 200) had not kept their retainers in the box provided during eating out, and lost them due as they were cleaned away or left behind wrapped on a tissue. Patients have to be reminded during the delivery of retainers to be extra careful while removing their retainers in eating areas and to place it carefully in the box provided. 19 retainers were lost as patients left their retainers in class / school. While a few orthodontist recommend not wearing the retainers to school, some still follow the full-time wear policy 6 months post debonding. Another finding of our study was the amount of retainers damaged by pets (mostly dogs) chewing on them. There is no scientific reason as to why the pets chewed on them but it could be due to the saliva in the retainers that attracts the pets and makes them chew on it thereby damaging it. Patients should be asked to keep their retainers away from the reach of their pets as it was the second most common reason for replacing retainers. Males tend to lose more retainers compared to females, the reasoning behind this could be the fact that girls tend to carry handbags and it is easier for them to carry the retainer box with them in their handbags. Patients need to understand the importance of wearing these retainers and replacing them on time in case any incident happens. Failure to do so can result in relapse as 16 of the patients in the study did not replace their removable retainers on time and returned with signs of relapse. While sometimes minor relapses can be managed with active retainers, some instances can also require retreatment (Table 2).

Table 2 Reason for lost/damage

\begin{tabular}{ll}
\hline Lost - Reason & No. of Patients \\
\hline In a restaurant/ food court & 113 \\
While swimming & 7 \\
Left it in class/school & 19 \\
Others & 32 \\
Damaged - Reason & \\
By pets & 21 \\
During travel & 6 \\
Others & 2 \\
\hline
\end{tabular}

\section{Conclusion}

We have based our conclusions on important tips and reminders that orthodontists need to inform patients on avoiding possible damage or losing their removable retainers:

a. Wear your removable retainers for the prescribed amount of time as instructed by your orthodontist. To prevent movement of teeth, it is very important that you wear it precisely as discussed.

b. You are not allowed to eat anything with your removable retainer in your mouth. When you remove your retainer for eating, remember to put them in the case provided and someplace safe. Do not put them in your lunch bag or wrap them in a napkin, as this is the most common way retainers are lost.

c. Keep your retainers away from your pets as this is the second 
most common way retainers are lost.

d. During travelling, always place them in a case and then in the suitcase, as this could also lead to potential damage.

e. Always remove your retainers for swimming or any contact sport.

f. Lastly, contact your orthodontist immediately if you misplace your retainers or if they are damaged.

\section{Funding}

None.

\section{Acknowledgments}

None.

\section{Conflicts of interest}

The author declares that there is no conflict of interest.

\section{References}

1. Johnston CD, Littlewood SJ. Retention in Orthodontics. Br Dent J. 2015;218(3):119-122.

2. Pratt MC, Kluemper GT, Hartsfield JK, et al. Evaluation of retention protocols among members of the American Association of Orthodontists in the United States. Am J Orthod Dentofacial Orthop. 2011;140(4):520 526.

3. Brezulier D, Turpin YL, Sorel O. A Protocol for treatment of minor orthodontic relapse during retention. $J$ Esthet Restor Dent. 2016;28(6):359-366. 